Published in final edited form as:

Eur J Nutr. 2017 March ; 56(2): 501-508. doi:10.1007/s00394-015-1095-7.

\title{
Plasma vitamin D biomarkers and leukocyte telomere length in men
}

\author{
Bettina Julin ${ }^{1,2,3}$, Irene M Shui ${ }^{4,5}$, Jennifer Prescott ${ }^{1,2}$, Edward L Giovannucci ${ }^{1,4,6}$, and \\ Immaculata De Vivo ${ }^{1,2,4}$ \\ ${ }^{1}$ Channing Division of Network Medicine, Department of Medicine, Brigham and Women's \\ Hospital and Harvard Medical School, Boston, MA \\ ${ }^{2}$ Program in Genetic Epidemiology and Statistical Genetics, Harvard T.H. Chan School of Public \\ Health, Boston, MA \\ ${ }^{3}$ Division of Nutritional Epidemiology, Institute of Environmental Medicine, Karolinska Institutet, \\ Stockholm, Sweden \\ ${ }^{4}$ Department of Epidemiology, Harvard T.H. Chan School of Public Health, Boston, MA \\ ${ }^{5}$ Division of Public Health Sciences, Fred Hutchinson Cancer Research Center, Seattle, \\ Washington \\ ${ }^{6}$ Department of Nutrition, Harvard T.H. Chan School of Public Health, Boston, MA
}

\begin{abstract}
Purpose-Vitamin D may reduce telomere shortening through anti-inflammatory and anti-cell proliferation mechanisms. In women, higher plasma 25-hydroxyvitamin D $(25(\mathrm{OH}) \mathrm{D})$ has been shown to be associated with longer telomere length, but the relationship has not been assessed in men.
\end{abstract}

Methods-We conducted a cross-sectional analysis of $25(\mathrm{OH}) \mathrm{D}, 1,25$-dihydroxyvitamin D $\left(1,25(\mathrm{OH})_{2} \mathrm{D}\right)$ and relative leukocyte telomere length (LTL) among 2,483 men (1,832 men for $\left.1,25(\mathrm{OH})_{2} \mathrm{D}\right)$ who were selected as cases and controls in three studies of telomeres and cancer nested within the Health Professionals Follow-up Study. We also genotyped 95 SNPs representing common genetic variation in vitamin D pathway genes. LTL was measured by quantitative PCR and z-scores within each study were calculated. Associations were assessed by linear as well as logistic regression adjusting for age and other potential confounders.

Results-Age $(P$-trend $<0.0001)$, pack-years of smoking $(P$-trend $=0.04)$ and body mass index $(P$-trend $=0.05)$ were inversely associated with LTL. Neither $25(\mathrm{OH}) \mathrm{D}$ or $1,25(\mathrm{OH})_{2} \mathrm{D}$ were

\footnotetext{
Corresponding author: Immaculata De Vivo, Channing Division of Network Medicine, Department of Medicine, Brigham and Women's Hospital and Harvard Medical School, Boston, MA 02115. Phone: 617-525-2094; Fax: 617-525-2008; nhidv@ channing.harvard.edu.

Ethical standards

This study was approved by the Human Subjects Committee of the Harvard School of Public Health (NIH/NCI R01 CA133891) and written informed consent was obtained from all participants.

Conflict of interest

The authors declare that they have no conflict of interest.
} 
associated with LTL (multivariable-adjusted P-trend 0.69 and 0.41, respectively, for the linear regression model). One SNP in the retinoid X receptor alpha gene was associated with long LTL $(P=0.0003)$.

Conclusions-In this cross-sectional study of men, $25(\mathrm{OH}) \mathrm{D}$ and $1,25(\mathrm{OH})_{2} \mathrm{D}$ were not associated with relative LTL.

\section{Keywords}

cross-sectional; men; telomeres; vitamin d; vitamin d pathway SNPs

\section{Introduction}

Vitamin D is mainly produced in the skin after exposure to sunlight. It is found naturally in foods such as fatty fish and fish liver oils and is fortified in milk and cereals. Vitamin D and its metabolites are primarily transported by the vitamin D-binding protein in the circulation. Vitamin D becomes biologically active through two hydroxylations; the first occurs in the liver and converts vitamin D to 25-hydroxyvitamin D (25(OH)D), the second hydroxylation takes place in various organs to form the physiologically active 1,25 -dihydroxyvitamin $\mathrm{D}$ $\left(1,25(\mathrm{OH})_{2} \mathrm{D}\right)$, which binds to the vitamin $\mathrm{D}$ receptor (VDR) in the cell nucleus.

Historically, vitamin D was known to play a main role in regulating calcium metabolism, but more recently, it has been shown to be involved in multiple cellular processes, including those that are cancer related, such as protecting cells against DNA damage, inducing cell cycle arrest and, consequently, inhibiting cell proliferation, increasing apoptosis and inducing differentiation [1,2]. Vitamin D deficiency is associated with increased incidence of cancer, cardiovascular disease, and other chronic illnesses, though there is still uncertainty over whether these associations are causal [3].

Telomeres consist of telomeric proteins and repetitive DNA sequences (TTAGGG) that protect the ends of linear chromosomes from attrition or from fusion with neighboring chromosomes and are thus important for maintaining chromosome stability. Standard DNA polymerase cannot synthesize the terminal $3^{\prime}$ end of template telomeric DNA during cell division, a phenomenon also called the end replication problem, which results in progressive telomere shortening. The enzyme complex telomerase replicates those sequences, keeping telomere length constant after each cellular division. However, since adult somatic cells typically have low or absent telomerase activity telomeres shorten over cell division in these cells. Therefore, telomere length in somatic cells reflects the cells' replicative history and can predict its remaining proliferative potential. Telomere shortening is accelerated by oxidative stress, inflammation, and cell proliferation [4-6] and shorter leukocyte telomeres have been associated with increased incidence of chronic diseases such as cancer [7] and cardiovascular disease [8].

Shorter telomere length has been associated with many chronic diseases that vitamin D protects against, thus it can be hypothesized that this protection may be mediated in part through the maintenance of telomere integrity. The presence of $1,25(\mathrm{OH})_{2} \mathrm{D}$ receptors in leukocytes, supports a direct effect of vitamin D on leukocyte telomere length [9]. Two recent studies found that higher plasma $25(\mathrm{OH}) \mathrm{D}$ concentration were significantly 
associated with longer leukocyte telomere length (LTL) in women [10,11]. To our knowledge, the association between vitamin $\mathrm{D}$ and telomere length in men remains unexplored. Since men in general have shorter telomeres than women [12], sex differences in the association between vitamin $\mathrm{D}$ and telomere length may be present.

In this cross-sectional study of a male cohort, the Health Professionals Follow-up Study (HPFS), we investigated concentrations of circulating $25(\mathrm{OH}) \mathrm{D}$ and $1,25(\mathrm{OH})_{2} \mathrm{D}$ as well as genetic variants of the vitamin D pathway in relation to telomere length in peripheral blood leukocytes. We also examined whether the association between circulating $25(\mathrm{OH}) \mathrm{D}$ and $1,25(\mathrm{OH})_{2} \mathrm{D}$ and telomere length was modified by total dietary calcium, folate, and retinol.

\section{Materials and Methods}

\section{Study population}

The HPFS cohort includes 51,529 US male health professionals who were aged 40-75 years at enrollment in 1986 when they responded to a questionnaire about their medical history and lifestyle. The men were mailed questionnaires to update this information every 2 years. Detailed dietary information was collected every 4 years. The overall follow-up rate was greater than 94\% [13]. Between 1993 and 1995, 18,018 cohort participants provided blood samples. Details on blood draw procedures, transportation, and storage of plasma samples in these cohorts have been described previously [14].

Multiple nested case-control studies have been conducted within the HPFS sub-cohort containing pre-diagnostic blood samples to investigate associations of vitamin D with for example prostate, colon and pancreatic cancer. The present analysis includes data on a total of 2,483 men from three of these nested case-control studies ( $n=225$ based on pancreatic cancer, $n=425$ based on colon cancer and $n=1,833$ based on prostate cancer), with previously measured $25(\mathrm{OH}) \mathrm{D}$, LTL and a completed food frequency questionnaire (FFQ) at the time of blood draw. In addition, 1,832 participants from the prostate nested case-control set had a measurement of $1,25(\mathrm{OH})_{2} \mathrm{D}$. The majority (95\%) of the HPFS blood cohort is white men of European ancestry; since both vitamin D concentrations and telomere length differ by race we restricted the analyses to white men.

This study was approved by the Human Subjects Committee of the Harvard School of Public Health (NIH/NCI R01 CA133891) and written informed consent was obtained from all participants.

\section{Measurement of vitamin D}

For the prostate and colon case-control sets, plasma $25(\mathrm{OH}) \mathrm{D}$ and $1,25(\mathrm{OH})_{2} \mathrm{D}$ measurements (prostate set only) were performed at the laboratory of Dr. Bruce W. Hollis at the Medical University of South Carolina using a radioimmunoassay method [15]. For the pancreatic case-control set, plasma concentrations of $25(\mathrm{OH}) \mathrm{D}$ were assayed in the laboratory of Dr. Nader Rifai (Children's Hospital, Boston, MA), using the 25Hydroxyvitamin D Enzyme Immunoassay Kit from Immunodiagnostic Systems. The comparability between the two methods was previously assessed in a separate pilot study (not published) where samples were first measured using the radioimmunoassay and then re- 
measured using the enzyme immunoassay. The values were highly comparable. The prostate cancer case-control samples were assayed for $25(\mathrm{OH}) \mathrm{D}$ in four different batches with mean intrapair coefficients of variation (CV) calculated from blinded quality control samples of $5.4 \%, 5.6 \%, 14.8 \%$, and $5.6 \%$ for batches 1 to 4 , respectively. For $1,25(\mathrm{OH})_{2} \mathrm{D}$, the CVs were $5.3 \%, 7.3 \%, 21.1 \%$ and $13.2 \%$, respectively. The colon and pancreatic cancer sets were assayed as one batch each with mean intrapair CVs of $10.1 \%$ and $\leq 9 \%$, respectively. Repeated blood samples on 144 individuals in the HPFS collected approximately 3 years apart yielded a Pearson correlation coefficient of 0.7 (adjusted for age, race, and season) for 25(OH)D [16]. To remove unwanted variability from laboratory, batch as well as season of blood draw, we standardized the 25(OH)D values to the average batch and season [17]. In brief, we regressed levels of $25(\mathrm{OH}) \mathrm{D}$ on indicator variables for each batch and season. Within each batch season combination, 25(OH)D levels were recalibrated by adding the resulting value of the coefficients for that batch minus the average of the batch coefficients. These recalibrated levels account for the variability between batch and season. For categorical 25(OH)D, we created season- and batch-specific cut points based on levels in the control subjects. Seasons were defined as summer (June to August), autumn (September to November), spring (March to May), and winter (December to February). Since $1,25(\mathrm{OH})_{2} \mathrm{D}$ does not vary with season, $1,25(\mathrm{OH})_{2} \mathrm{D}$ values were only adjusted for batch as described above.

\section{Covariate assessment}

Intakes of vitamin $\mathrm{D}$, calcium, folate and retinol were calculated using the information from the FFQ closest to the time the blood sample was provided (1994 FFQ) or, if not available, from the most recent questionnaire administered before 1994. Similarly, we used information from the 1994 questionnaire or, if not available, the most recent before 1994 to estimate BMI, smoking amount (indicated by pack-years), alcohol consumption and physical activity (indicated by metabolic equivalent per week; MET/week) at the time of blood donation.

\section{Single-nucleotide polymorphisms (SNP) selection and genotyping}

The SNP selection has been described in detailed elsewhere [18]. Briefly, 97 tagging SNPs $\left(\mathrm{R}^{2}>0.8\right)$ were selected from seven vitamin D-related genes ( VDR, CYP27B1, GC, $C Y P 27 A 1, C Y P 2 R 1, C Y P 24 A 1$ and $R X R A)$. Selection was restricted to those with a minor allele frequency of greater than 5\% in the reference panel. One SNP in $V D R$ and one in CYP24A1 failed genotyping. Blood samples from matched case-control pairs were handled identically and assayed in the same batch in a blinded fashion. Genotyping was performed at the Dana Farber/Harvard Cancer Center High-Throughput Genotyping Core using the TaqMan Open Array (Applied Biosystems, Foster City, CA). Blinded quality control samples were inserted to validate genotyping procedures. All SNPs had greater than $90 \%$ genotype completion, and the concordance was greater than $99 \%$ for blinded quality control samples. Participants with missing values had their genotypes imputed using the HapMap Phase III CEU data and the MACH imputation program [19]. 


\section{Telomere length assay}

Genomic DNA was extracted from peripheral blood leukocytes using the QIAmp 96-spin blood protocol (Qiagen, Chatsworth, CA, USA). Pico-Green quantification of genomic DNA was performed using a Molecular Devices 96-well spectrophotometer (Sunnyvale, CA, USA). Relative LTL was determined using a modified, high-throughput version of the quantitative PCR (qPCR) based telomere assay. The qPCR telomere assay was run on Applied Biosystems 7900HT Sequence Detection System (Foster City, CA, USA). Laboratory personnel were blinded to participant characteristics and all assays were processed in triplicates by the same technician, and under identical conditions. The average relative LTL was calculated as the ratio of telomere repeat copy number to a single gene (36B4) copy number (T/S). Relative LTL is reported as the exponentiated T/S ratio corrected for a reference sample included on every plate. In all three nested case-control studies, the telomere and single-gene assay CVs for triplicates were less than $1.2 \%$. CVs for the exponential $\mathrm{T} / \mathrm{S}$ ratio ranged from $11.8-16.0 \%$. Although this assay provides a relative measurement of telomere length, T/S ratios highly correlate with absolute telomere lengths determined by Southern blot $(r=0.82 ; \mathrm{p}<0.001)$ [20].

Telomere length was assayed in study-specific batches. To minimize the impact of a potential batch effect, z-scores of log-transformed LTL were calculated by standardizing the LTL in comparison to the mean within each individual study.

\section{Statistical analyses}

We detected and excluded two outliers based on the log-normalized LTL in the pancreas dataset using the generalized extreme studentized deviate (ESD) test [21]; both were on the short side of the distribution. No outliers were detected in the colon and prostate datasets. Linear regression was used when the telomere length $z$-score outcome was continuous, and unconditional logistic regression (odds ratios and $95 \%$ confidence intervals) was used when the $z$-score was dichotomized at the median. We adjusted for age (continuous, years), smoking $\left(0,>0-20,>20-40,>40\right.$ pack-years), BMI (continuous, $\left.\mathrm{kg} / \mathrm{m}^{2}\right)$, physical activity (continuous, MET/week) and alcohol consumption (continuous, g/day) as potential confounders since these factors have previously been associated with vitamin $\mathrm{D}$ and/or telomere length. Missing values of smoking (around 2\%) where treated as a separate missing category. Multiplicative interaction terms involving continuous variables were created to test for effect modification using the Wald test.

The additive genetic model was used for the SNP analyses, which assumes that the effect of the heterozygous genotype is intermediate between the two homozygous genotypes. The homozygous genotype of the reference allele was coded as 0 . Age-adjusted linear regression of each individual SNP and continuous LTL as well as unconditional logistic regression with low and high LTL (dichotomized at the median) were performed. The 95 SNPs corresponded to 89 independent tests; consequently, a P value significance threshold of 0.0006 controls the experiment-wide type I error rate at the 0.05 level. In addition, we assessed global associations for SNP sets defined by all seven genes as well as each gene individually using a logistic kernel machine model, described in detail in Shui et al [18]. All 
$P$-values were two-sided and analyses were performed using SAS Version 9.2 software (SAS Institute, Cary, NC) or R (http://www.r-project.org/).

\section{Results}

Main age-standardized characteristics of the study population by quartiles of telomere length are presented in Table 1. As expected, there was an inverse correlation between age at blood draw and telomere length: younger men had longer telomeres $(P$-trend $<0.0001)$. Moreover, men with longer telomeres smoked slightly less $(P$-trend $=0.04)$ and had lower BMI $(P$ trend $=0.05)$ than men with shorter telomeres.

In Table 2, associations between key variables and plasma 25(OH)D are reported. Men with higher plasma 25(OH)D concentrations had lower BMI $(P$-trend $<0.0001)$ and smoked less $(P$-trend 0.01$)$ than men with lower concentrations, whereas physical activity $(P$-trend $<0.0001$ ), alcohol consumption ( $P$-trend $<0.0001$ ), calcium, folate, retinol and total dietary vitamin D intake, as well as plasma $1,25(\mathrm{OH})_{2} \mathrm{D}$ all showed a positive association with plasma 25(OH)D $(P$-trend $=0.0002$ or lower for all $)$.

As depicted in Table 3, we did not observe an association between plasma vitamin D biomarkers and LTL $z$-scores, neither in age-adjusted $(P$-trend $=0.97$ for 25(OH)D and 0.34 for $\left.1,25(\mathrm{OH})_{2} \mathrm{D}\right)$ nor multivariable-adjusted models $(P$-trend $=0.69$ for $25(\mathrm{OH}) \mathrm{D}$ and 0.41 for $\left.1,25(\mathrm{OH})_{2} \mathrm{D}\right)$. We also performed sensitivity analyses, excluding individuals who later developed cancer. Results did not differ in those analyses restricted to controls; multivariable-adjusted $P$-trend $=0.72$ for $25(\mathrm{OH}) \mathrm{D}(\mathrm{n}=1,356$ of whom $\mathrm{n}=162$ were from the pancreatic cancer set, $n=279$ were from the colon cancer set and $n=915$ were from the prostate cancer set) and 0.75 for $1,25(\mathrm{OH})_{2} \mathrm{D}(\mathrm{n}=915$ from the prostate cancer set). Logistic regression analyses did not indicate a dose-response relationship between either 25(OH)D or $1,25(\mathrm{OH})_{2} \mathrm{D}$ and LTL z-scores in all participants (Table 4) or in analyses restricted to healthy men (data not shown). Our assessment of whether calcium, folate or retinol modified the association between plasma biomarkers of vitamin $\mathrm{D}\left(25(\mathrm{OH}) \mathrm{D}\right.$ and $\left.1,25(\mathrm{OH})_{2} \mathrm{D}\right)$ and LTL showed no significant interactions. Moreover, we did not find an interaction with age.

A total of 1,833 individuals from the prostate nested case-control set were included in the genetic analyses of 95 SNPs. Figure 1 shows the per-allele log odds ratio for each SNP and telomere length (dichotomized by the median). Only rs41400444 (retinoid X receptor alpha; $R X R A$ ) showed a positive association after correcting for multiple testing (per-allele OR 1.67, 95\% CI: $1.26-2.19, P$-trend $=0.0003$; Supplemental Table S1). Five additional SNPs in $V D R, C Y P 24 A 1$ and $R X R A$ were nominally associated with long telomere length $(P<0.05)$. In the global pathway analyses, we did not observe an association between the total pathway SNP set and telomere length $(P=0.41)$ or any SNP sets defined by specific genes (Supplemental table S1). Assessing the association between the SNPs and continuous LTL z-score did not change the results considerably.

\section{Discussion}

In this cross-sectional study of white men, we did not observe an association between vitamin D biomarkers $\left(25(\mathrm{OH}) \mathrm{D}\right.$ and $\left.1,25(\mathrm{OH})_{2} \mathrm{D}\right)$ and leukocyte telomere length. The lack 
of association remained when restricting the analyses to healthy men. One SNP in the $R X R A$ gene showed a statistically significant positive association with long telomere length. To the best of our knowledge, there are no other studies published to date exploring the relation between vitamin $\mathrm{D}$, genes in the vitamin $\mathrm{D}$ pathway and telomere length in white men.

Consistent with other studies [7,10,22-27], we found that BMI and smoking were inversely associated with telomere length. Due to their G-rich nucleotide sequence, telomeres are highly sensitive to oxidative stress [28], which together with inflammation are probable mechanisms mediating the effects of BMI and smoking on telomere length [29,30]. Two previous cross-sectional studies in women suggest a modest, but significantly positive association between $25(\mathrm{OH}) \mathrm{D}$ and telomere length [10,11]. A possible explanation for the lack of an association in the present study is the relatively old age of the participants. For example, the study by Richards et al. which found an association between $25(\mathrm{OH}) \mathrm{D}$ and telomere length included much younger women (aged18-80 years, mean age $=49.4$ years), whereas the age range in our study was $46-81$ years, mean age $=64.1$ years. However, the study by Liu et al. was conducted in women of a similar age range (43-68 years, mean age $=59.4$ years) and did observe a positive, although modest association. Since men, in general, have shorter telomeres than women [12] we cannot exclude a real sex difference in the association between vitamin $\mathrm{D}$ and telomere length. Several biologically plausible explanations for a difference between men and women have been suggested. First, estrogen can stimulate the production of telomerase and, as opposed to testosterone, can reduce the presence of reactive oxygen species [31]. Second, since males are heterogametic (XY) any flaw in telomere maintenance alleles of the unguarded $\mathrm{X}$ chromosome may give rise to shorter telomeres [32].

One individual SNP (rs41400444) in the $R X R A$ gene was associated with longer telomere length. The $1,25(\mathrm{OH})_{2} \mathrm{D}-\mathrm{VDR}$ complex forms a heterodimer with the $R X R A$ in the cell nucleus before attaching to target genes [33], but $R X R$ has other roles in the cell that are independent of the vitamin D pathway [34]. Therefore the association between one common variant and long telomere length does not necessarily imply a link between the vitamin D pathway and telomere length. Moreover, this finding should be interpreted with caution given the high number of tests. The strength of our study includes a large sample size and it is the first study to assess the association between biomarkers of vitamin $\mathrm{D}(25(\mathrm{OH}) \mathrm{D}$ and $\left.1,25(\mathrm{OH})_{2} \mathrm{D}\right)$, vitamin D-related genes and telomere length in men. There are however several limitations. Both $25(\mathrm{OH}) \mathrm{D}, 1,25(\mathrm{OH})_{2} \mathrm{D}$ and telomere length was only measured once and at the same time point. This means the measurements of $25(\mathrm{OH}) \mathrm{D}$ and $1,25(\mathrm{OH})_{2} \mathrm{D}$ may not reflect the biologically relevant concentration of exposure. With the cross-sectional design; we could not assess the impact of change in vitamin D concentration on telomere attrition rate. A longitudinal study with repeated measurements would provide a more comprehensive view of the effect on leukocyte telomere dynamics. The detection of absolute levels of $25(\mathrm{OH}) \mathrm{D}$ are controversial and may differ by assay. Thus, because the 25(OH)D assays differed across cohorts and were not calibrated to a common gold standard, we were unable to assess absolute cut-points for $25(\mathrm{OH}) \mathrm{D}$. However the, relative rankings adjusting for batch and season used in our analysis should reasonably robust. Because the study was conducted in men of European ancestry and it is known that vitamin D 
concentrations and telomere dynamics vary by ethnicity, the results may not be generalizable to other ethnicities. Finally, although all analyses were adjusted for multiple covariates, unmeasured or residual confounding could still be present. Since we did not observe evidence of strong confounding it is, however, unlikely that unmeasured confounding alone would explain the lack of an observed association.

In conclusion, this study did not show an association between biomarkers of vitamin D $\left(25(\mathrm{OH}) \mathrm{D}\right.$ and $\left.1,25(\mathrm{OH})_{2} \mathrm{D}\right)$ and telomere length. Since higher plasma $25(\mathrm{OH}) \mathrm{D}$ concentrations have been shown to be associated with longer telomere length in women, a sex difference in the association cannot be excluded.

\section{Supplementary Material}

Refer to Web version on PubMed Central for supplementary material.

\section{Acknowledgments}

The authors would like to thank the participants and staff of the Health Professionals Follow-up Study for their valuable contributions as well as the following state cancer registries for their help: AL, AZ, AR, CA, CO, CT, DE, FL, GA, ID, IL, IN, IA, KY, LA, ME, MD, MA, MI, NE, NH, NJ, NY, NC, ND, OH, OK, OR, PA, RI, SC, TN, TX, VA, WA, WY. The authors would also like to thank Channing Division of Network Medicine, Department of Medicine, Brigham and Women's Hospital and Harvard Medical School and in addition Pati Soule, Esther Orr and Hardeep Ranu for their laboratory assistance.

The Health Professionals Follow-up Study is supported by an infrastructure grant from the National Institutes of Health/National Cancer Institute (UM1 CA167552). BJ is also supported by a grant from the Swedish Research Council for Health, Working Life and Welfare; IMS is supported by a Department of Defense Prostate Cancer Research Program fellowship; IDV is supported by grant R01 CA082838 and ELG is supported by grant R01 CA133891 from the National Cancer Institute.

\section{Abbreviations}

25(OH)D

$1,25(\mathrm{OH}) 2 \mathrm{D}$

CV

FFQ

HPFS

LTL

MET

qPCR

SNP

VDR 25-hydroxyvitamin D

1,25-dihydroxyvitamin D

coefficients of variation

food frequency questionnaire

Health Professionals Follow-up Study

leukocyte telomere length

metabolic equivalent per week

quantitative PCR

Single-nucleotide polymorphism

vitamin D receptor 


\section{References}

1. Deeb KK, Trump DL, Johnson CS. Vitamin D signalling pathways in cancer: potential for anticancer therapeutics. Nature reviews Cancer. 2007; 7(9):684-700. DOI: 10.1038/nrc2196 [PubMed: 17721433]

2. Nagpal S, Na S, Rathnachalam R. Noncalcemic actions of vitamin D receptor ligands. Endocrine reviews. 2005; 26(5):662-687. DOI: 10.1210/er.2004-0002 [PubMed: 15798098]

3. Holick MF. Vitamin D deficiency. The New England journal of medicine. 2007; 357(3):266-281. DOI: 10.1056/NEJMra070553 [PubMed: 17634462]

4. Artandi SE, DePinho RA. Telomeres and telomerase in cancer. Carcinogenesis. 2010; 31(1):9-18. DOI: 10.1093/carcin/bgp268 [PubMed: 19887512]

5. Houben JM, Moonen HJ, van Schooten FJ, Hageman GJ. Telomere length assessment: biomarker of chronic oxidative stress? Free radical biology \& medicine. 2008; 44(3):235-246. DOI: 10.1016/ j.freeradbiomed.2007.10.001 [PubMed: 18021748]

6. von Zglinicki T. Role of oxidative stress in telomere length regulation and replicative senescence. Annals of the New York Academy of Sciences. 2000; 908:99-110. [PubMed: 10911951]

7. Prescott J, McGrath M, Lee IM, Buring JE, De Vivo I. Telomere length and genetic analyses in population-based studies of endometrial cancer risk. Cancer. 2010; 116(18):4275-4282. DOI: 10.1002/cncr.25328 [PubMed: 20549820]

8. Butt HZ, Atturu G, London NJ, Sayers RD, Bown MJ. Telomere length dynamics in vascular disease: a review. European journal of vascular and endovascular surgery : the official journal of the European Society for Vascular Surgery. 2010; 40(1):17-26. DOI: 10.1016/j.ejvs.2010.04.012

9. Provvedini DM, Tsoukas CD, Deftos LJ, Manolagas SC. 1,25-dihydroxyvitamin D3 receptors in human leukocytes. Science (New York, NY). 1983; 221(4616):1181-1183.

10. Liu JJ, Prescott J, Giovannucci E, Hankinson SE, Rosner B, Han J, De Vivo I. Plasma vitamin D biomarkers and leukocyte telomere length. American journal of epidemiology. 2013; 177(12): 1411-1417. DOI: 10.1093/aje/kws435 [PubMed: 23660800]

11. Richards JB, Valdes AM, Gardner JP, Paximadas D, Kimura M, Nessa A, Lu X, Surdulescu GL, Swaminathan R, Spector TD, Aviv A. Higher serum vitamin D concentrations are associated with longer leukocyte telomere length in women. The American journal of clinical nutrition. 2007; 86(5):1420-1425. [PubMed: 17991655]

12. Gardner M, Bann D, Wiley L, Cooper R, Hardy R, Nitsch D, Martin-Ruiz C, Shiels P, Sayer AA, Barbieri M, Bekaert S, Bischoff C, Brooks-Wilson A, Chen W, Cooper C, Christensen K, De Meyer T, Deary I, Der G, Diez Roux A, Fitzpatrick A, Hajat A, Halaschek-Wiener J, Harris S, Hunt SC, Jagger C, Jeon HS, Kaplan R, Kimura M, Lansdorp P, Li C, Maeda T, Mangino M, Nawrot TS, Nilsson P, Nordfjall K, Paolisso G, Ren F, Riabowol K, Robertson T, Roos G, Staessen JA, Spector T, Tang N, Unryn B, van der Harst P, Woo J, Xing C, Yadegarfar ME, Park JY, Young N, Kuh D, von Zglinicki T, Ben-Shlomo Y. Gender and telomere length: systematic review and meta-analysis. Experimental gerontology. 2014; 51:15-27. DOI: 10.1016/j.exger.2013.12.004 [PubMed: 24365661]

13. Rimm EB, Stampfer MJ, Colditz GA, Giovannucci E, Willett WC. Effectiveness of various mailing strategies among nonrespondents in a prospective cohort study. American journal of epidemiology. 1990; 131(6):1068-1071. [PubMed: 2343859]

14. Wei EK, Giovannucci E, Fuchs CS, Willett WC, Mantzoros CS. Low plasma adiponectin levels and risk of colorectal cancer in men: a prospective study. Journal of the National Cancer Institute. 2005; 97(22):1688-1694. DOI: 10.1093/jnci/dji376 [PubMed: 16288122]

15. Hollis BW. Quantitation of 25-hydroxyvitamin D and 1,25-dihydroxyvitamin D by radioimmunoassay using radioiodinated tracers. Methods in enzymology. 1997; 282:174-186. [PubMed: 9330287]

16. Platz EA, Leitzmann MF, Hollis BW, Willett WC, Giovannucci E. Plasma 1,25-dihydroxy- and 25hydroxyvitamin D and subsequent risk of prostate cancer. Cancer causes \& control : CCC. 2004; 15(3):255-265. DOI: 10.1023/B:CACO.0000024245.24880.8a [PubMed: 15090720] 
17. Rosner B, Cook N, Portman R, Daniels S, Falkner B. Determination of blood pressure percentiles in normal-weight children: some methodological issues. American journal of epidemiology. 2008; 167(6):653-666. DOI: 10.1093/aje/kwm348 [PubMed: 18230679]

18. Shui IM, Mucci LA, Kraft P, Tamimi RM, Lindstrom S, Penney KL, Nimptsch K, Hollis BW, Dupre N, Platz EA, Stampfer MJ, Giovannucci E. Vitamin D-related genetic variation, plasma vitamin D, and risk of lethal prostate cancer: a prospective nested case-control study. Journal of the National Cancer Institute. 2012; 104(9):690-699. DOI: 10.1093/jnci/djs189 [PubMed: 22499501]

19. Li M, Byth K, Eastman CJ. Childhood overweight and obesity by socio-economic indexes for areas. The Medical journal of Australia. 2007; 187(3):195.

20. Cawthon RM. Telomere measurement by quantitative PCR. Nucleic acids research. 2002; 30(10):e47. [PubMed: 12000852]

21. Rosner B. Percentage Point for a Generalized ESD Many-Outlier Procedure. Technometrics. 1983; 25:165-172.

22. Aviv A, Chen W, Gardner JP, Kimura M, Brimacombe M, Cao X, Srinivasan SR, Berenson GS. Leukocyte telomere dynamics: longitudinal findings among young adults in the Bogalusa Heart Study. American journal of epidemiology. 2009; 169(3):323-329. DOI: 10.1093/aje/kwn338 [PubMed: 19056834]

23. McGrath M, Wong JY, Michaud D, Hunter DJ, De Vivo I. Telomere length, cigarette smoking, and bladder cancer risk in men and women. Cancer epidemiology, biomarkers \& prevention : a publication of the American Association for Cancer Research, cosponsored by the American Society of Preventive Oncology. 2007; 16(4):815-819. DOI: 10.1158/1055-9965.epi-06-0961

24. Nawrot TS, Staessen JA, Gardner JP, Aviv A. Telomere length and possible link to X chromosome. Lancet. 2004; 363(9408):507-510. DOI: 10.1016/s0140-6736(04)15535-9 [PubMed: 14975611]

25. Sun Q, Shi L, Prescott J, Chiuve SE, Hu FB, De Vivo I, Stampfer MJ, Franks PW, Manson JE, Rexrode KM. Healthy lifestyle and leukocyte telomere length in U.S. women. PloS one. 2012; 7(5):e38374.doi: 10.1371/journal.pone.0038374 [PubMed: 22675460]

26. Valdes AM, Andrew T, Gardner JP, Kimura M, Oelsner E, Cherkas LF, Aviv A, Spector TD. Obesity, cigarette smoking, and telomere length in women. Lancet. 2005; 366(9486):662-664. DOI: 10.1016/s0140-6736(05)66630-5 [PubMed: 16112303]

27. Zannolli R, Mohn A, Buoni S, Pietrobelli A, Messina M, Chiarelli F, Miracco C. Telomere length and obesity. Acta paediatrica (Oslo, Norway : 1992). 2008; 97(7):952-954. DOI: 10.1111/j. 1651-2227.2008.00783.x

28. Henle ES, Han Z, Tang N, Rai P, Luo Y, Linn S. Sequence-specific DNA cleavage by Fe2+mediated fenton reactions has possible biological implications. The Journal of biological chemistry. 1999; 274(2):962-971. [PubMed: 9873038]

29. Aviv A. Leukocyte telomere length: the telomere tale continues. The American journal of clinical nutrition. 2009; 89(6):1721-1722. DOI: 10.3945/ajcn.2009.27807 [PubMed: 19386739]

30. von Zglinicki T. Oxidative stress shortens telomeres. Trends in biochemical sciences. 2002; 27(7): 339-344. [PubMed: 12114022]

31. Aviv A. Telomeres, sex, reactive oxygen species, and human cardiovascular aging. Journal of molecular medicine (Berlin, Germany). 2002; 80(11):689-695. DOI: 10.1007/s00109-002-0377-8

32. Barrett EL, Richardson DS. Sex differences in telomeres and lifespan. Aging cell. 2011; 10(6): 913-921. DOI: 10.1111/j.1474-9726.2011.00741.x [PubMed: 21902801]

33. Giovannucci E. The epidemiology of vitamin D and cancer incidence and mortality: a review (United States). Cancer causes \& control : CCC. 2005; 16(2):83-95. DOI: 10.1007/ s10552-004-1661-4 [PubMed: 15868450]

34. Dawson MI, Xia Z. The retinoid X receptors and their ligands. Biochimica et biophysica acta. 2012; 1821(1):21-56. DOI: 10.1016/j.bbalip.2011.09.014 [PubMed: 22020178] 


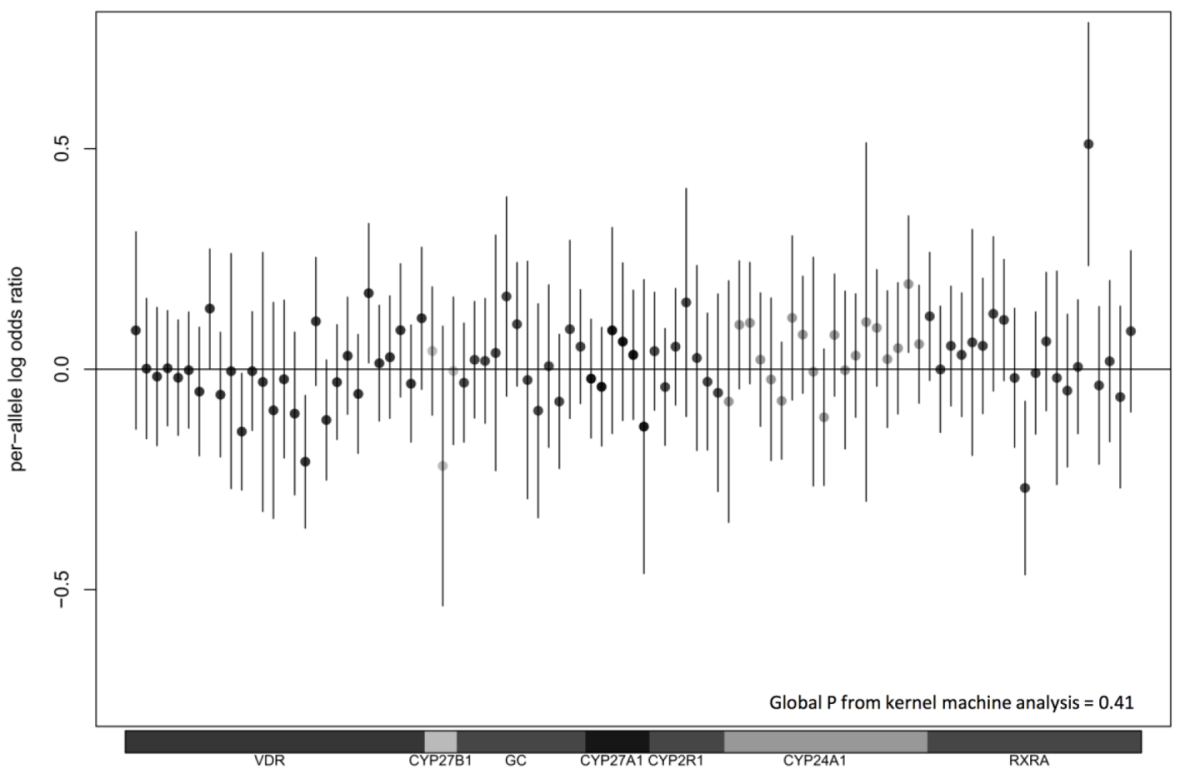

Fig. 1.

Per-allele log odds ratio for each single-nucleotide polymorphism (SNP) and long relative leukocyte telomere length (above the median), $n=1,833$. Each dot represents a SNP and is presented by gene 


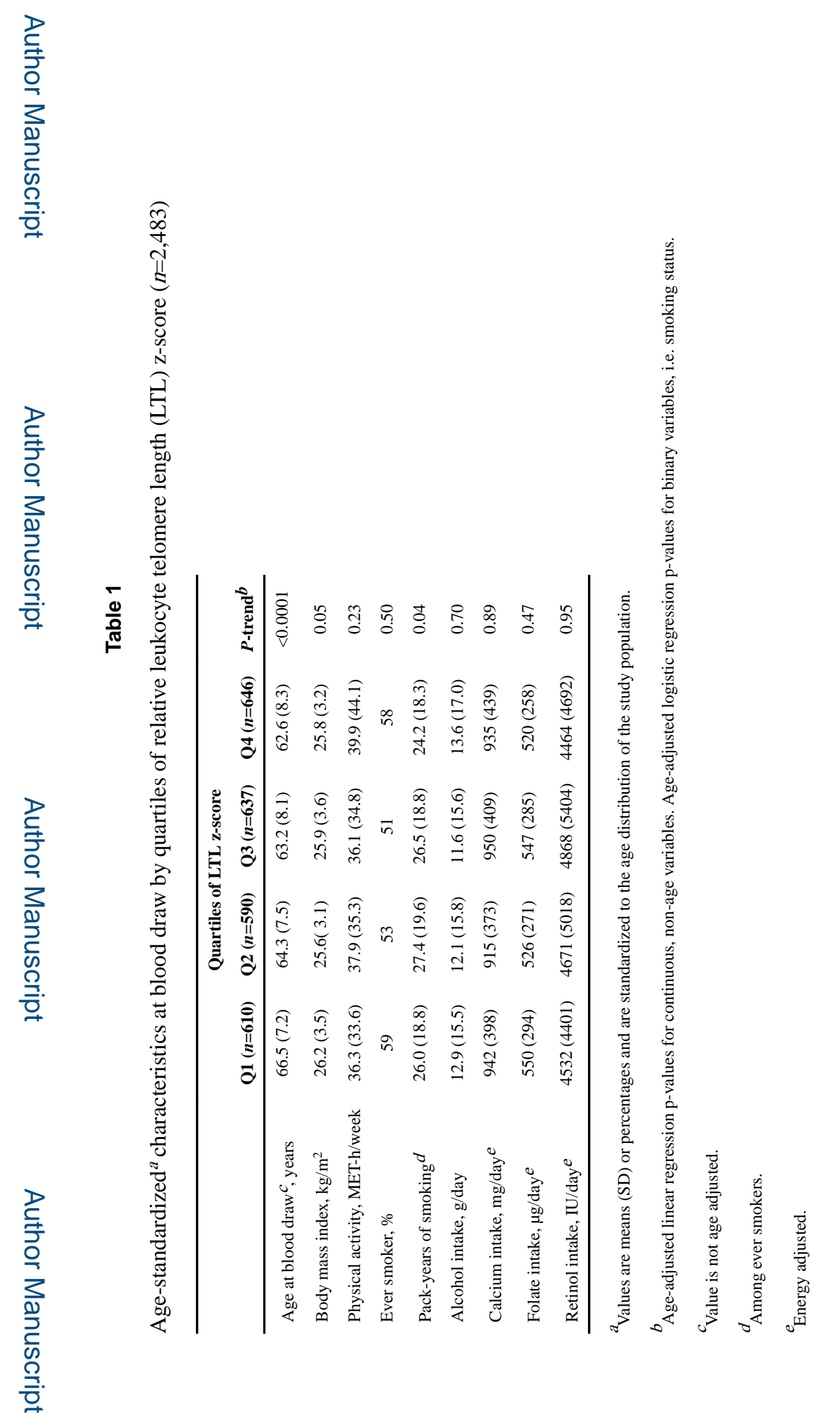

Eur J Nutr. Author manuscript; available in PMC 2018 March 01. 


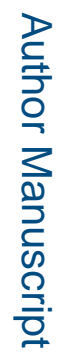

D্

@)

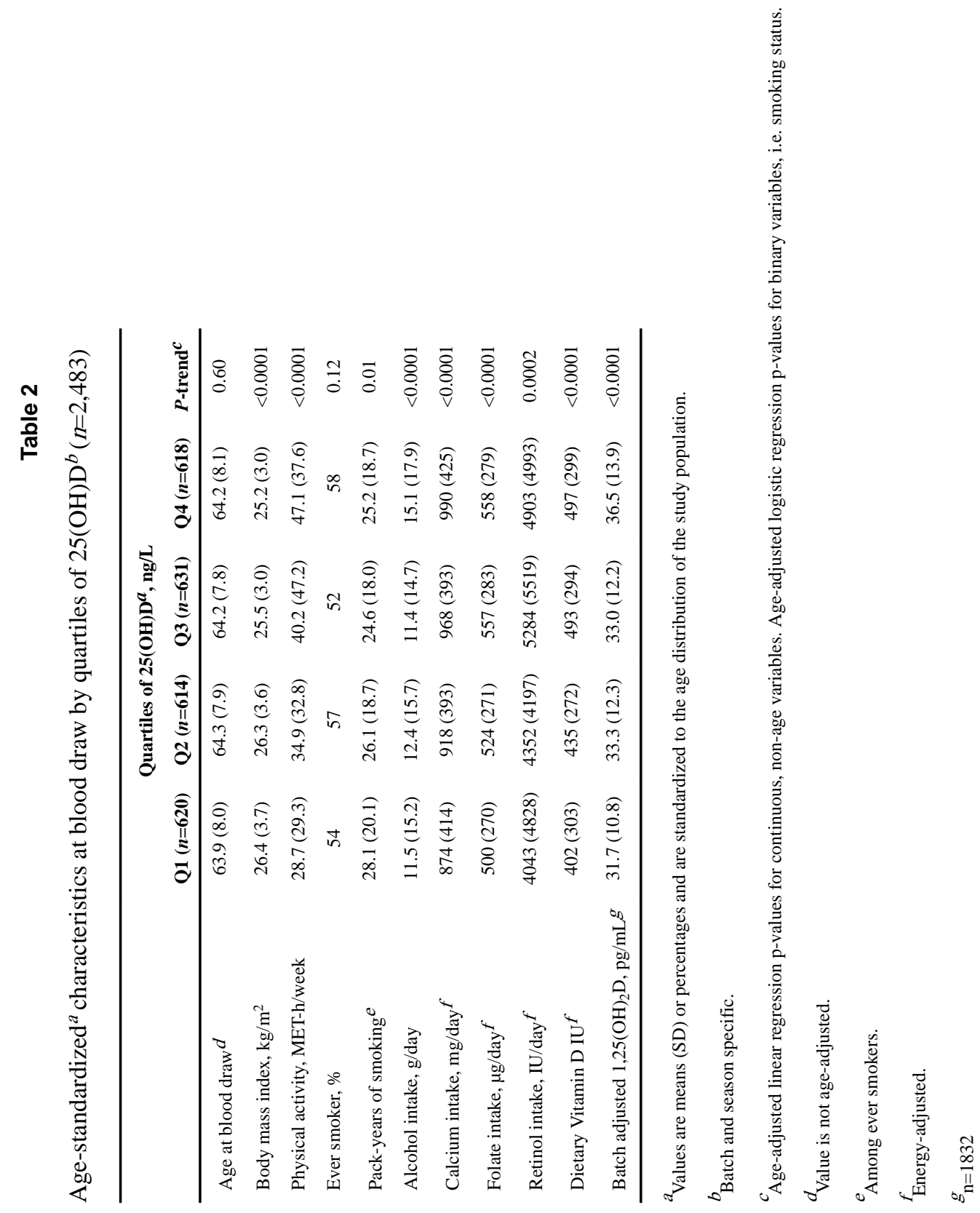

Eur J Nutr. Author manuscript; available in PMC 2018 March 01. 

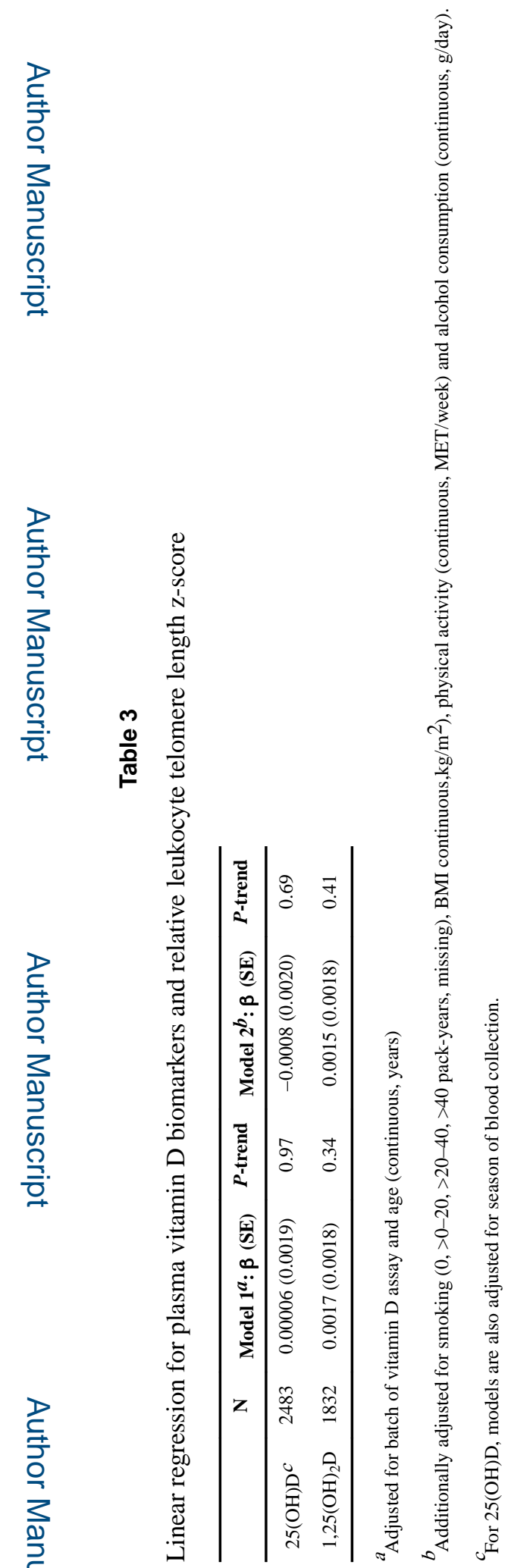

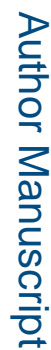

Eur J Nutr. Author manuscript; available in PMC 2018 March 01. 

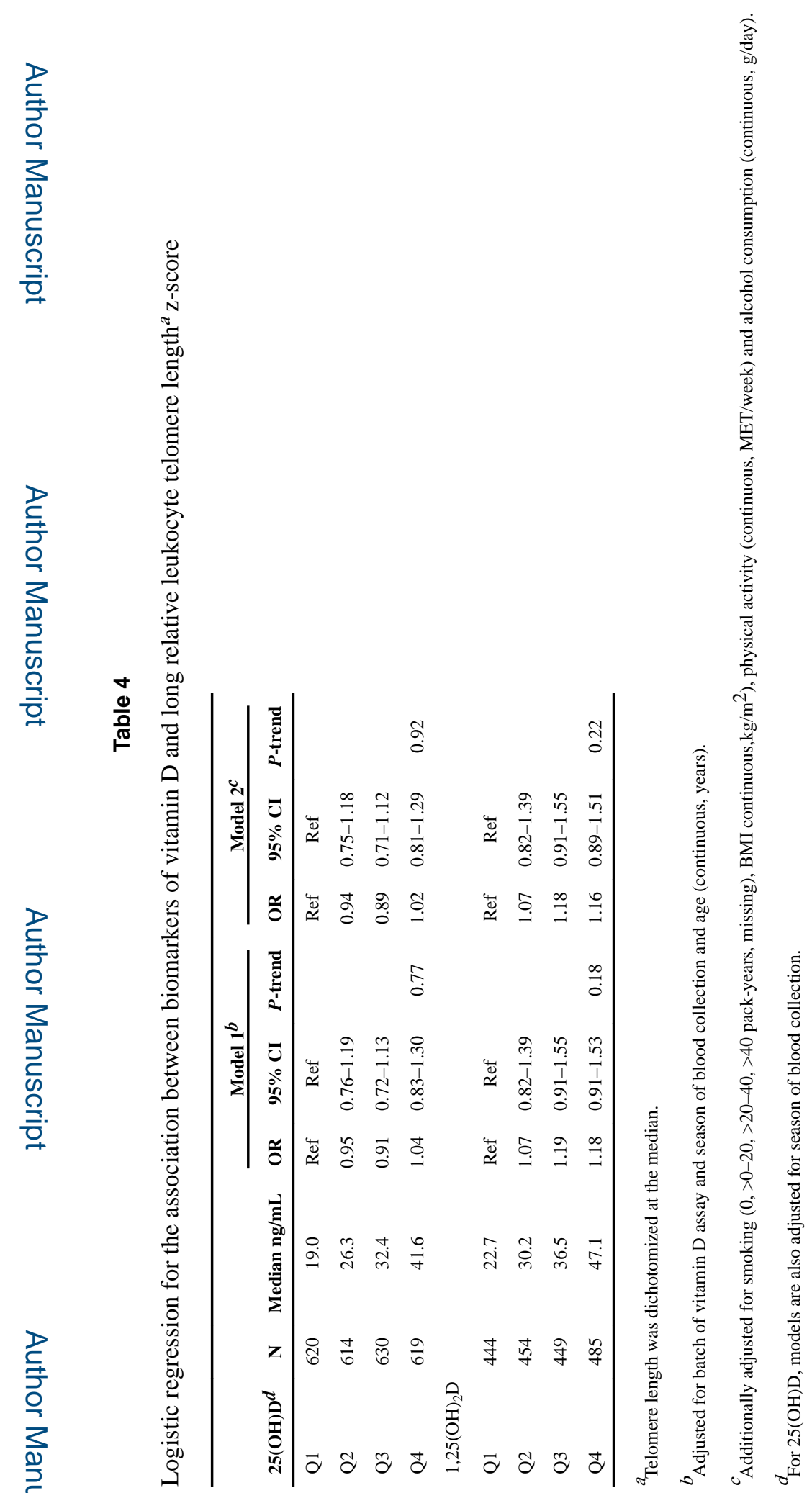

Eur J Nutr. Author manuscript; available in PMC 2018 March 01. 